\title{
A Review of an Expert System Design for Crude Oil Distillation Column Using the Neural Networks Model and Process Optimization and Control Using Genetic Algorithm Framework
}

\author{
Lekan Taofeek Popoola1, Gutti Babagana ${ }^{2}$, Alfred Akpoveta Susu ${ }^{3}$ \\ ${ }^{1}$ Department of Petroleum and Chemical Engineering, Afe Babalola University, Ado-Ekiti, Nigeria \\ ${ }^{2}$ Department of Chemical Engineering, University of Maiduguri, Maiduguri, Nigeria \\ ${ }^{3}$ Department of Chemical Engineering, University of Lagos, Lagos, Nigeria \\ Email: poplektaoy27@yahoo.com, babaganagutti@yahoo.com, alfredasusu22@hotmail.com
}

Received January 11, 2013; revised March 11, 2013; accepted April 11, 2013

Copyright (C) 2013 Lekan Taofeek Popoola et al. This is an open access article distributed under the Creative Commons Attribution License, which permits unrestricted use, distribution, and reproduction in any medium, provided the original work is properly cited.

\begin{abstract}
This paper presents a comprehensive review of various traditional systems of crude oil distillation column design, modeling, simulation, optimization and control methods. Artificial neural network (ANN), fuzzy logic (FL) and genetic algorithm (GA) framework were chosen as the best methodologies for design, optimization and control of crude oil distillation column. It was discovered that many past researchers used rigorous simulations which led to convergence problems that were time consuming. The use of dynamic mathematical models was also challenging as these models were also time dependent. The proposed methodologies use back-propagation algorithm to replace the convergence problem using error minimal method.
\end{abstract}

Keywords: Artificial Neural Network; Crude Oil Distillation Column; Genetic Algorithm Framework; Sigmoidal Transfer Function; Back-Propagation Algorithm

\section{Introduction}

Crude oil distillation is the separation of hydrocarbons in crude oil into fractions based on their boiling points which lie within a specified range [1]. The separation is done in a large tower that is operated at atmospheric pressure. The tower contains a number of trays where hydrocarbon gases and liquids interact. The liquids flow down the tower and the gases up [2]. The fractions that rise highest in the column before condensing are called light fractions, and those that condense on the lowest trays are called heavy fractions [3]. Figure 1 gives a schematic representation of the process overview of a crude oil distillation column. This crude oil distillation column can be designed using artificial neural network. An artificial neural network (ANN) is an information processing paradigm that is inspired by the way biological nervous systems (such as the brain) process information. It is composed of a large number of highly interconnected processing elements (neurons) working in unison to solve specific problems [4] (Figure 2).
Artificial neural networks initially grew from the full understanding of some ideas and aspects about how biological systems work, especially the human brain. In biology, the cell body of neuron is called the soma. The spine-like extensions of the cell body are dendrites. They usually branch repeatedly and form a bushy tree around the cell body and provide connections to receive incomeing signals from other neurons. The axon extends away from the cell body to provide a pathway for outgoing signals. Signals are transferred from one neuron to another through a contact point called a synapse [3]. Like biological neuron networks, ANN is made of neuron and synaptic connections which are highly simplified abstracts of their counterparts in real neural networks (Figure 3). The neurons in the ANN are usually called units, node, or processing elements, and the efficacy of the synaptic connection, which is a measurement of excitability and inhabitability, are usually called weights. Neural networks systems (NNS) are typically organized in layers. Layers are made up of a number of interconnected nodes (artificial neurons or processing element), 


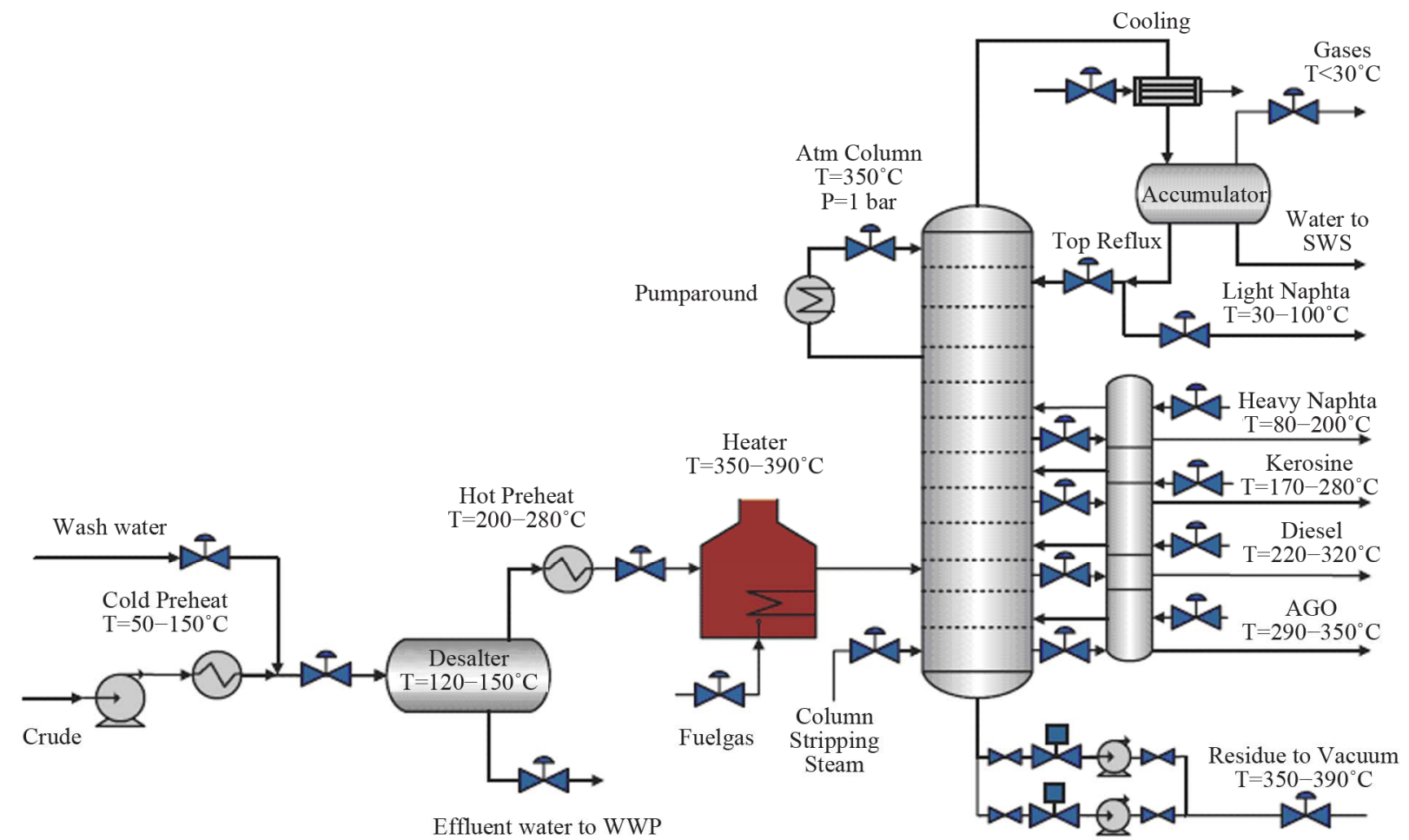

Figure 1. Process overview of crude oil distillation column.

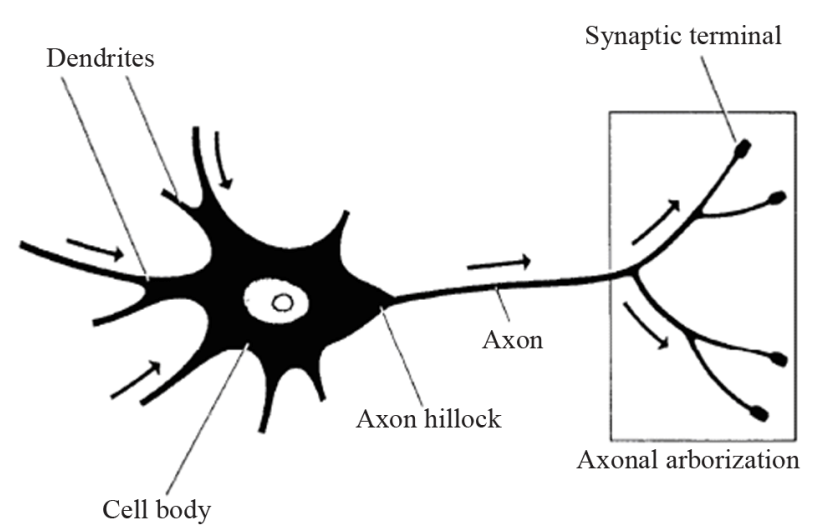

Figure 2. Basic neuron.

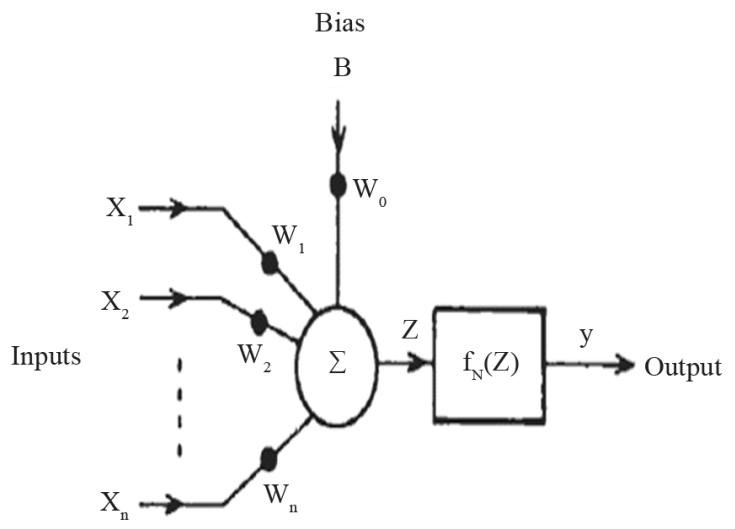

Figure 3. A biased neuron. which contains an activation function. The data are presented to the networks via the input layer, which communicates to one or more hidden layers where the actual processing is done through a system of weighted connections. The hidden layers are then linked to an output layer, which generates the output $[5,6]$.

The objective of crude oil distillation unit is to perform a process optimization including high production rate with required product quality and low operating costs by searching an optimal operating condition of the operating variables [7]. Genetic algorithm and fuzzy logic are adaptive methods which can be used to solve optimization problems. Genetic Algorithms are based on the principle of genetic processes of biological organisms while Fuzzy logic utilizes human knowledge by giving the fuzzy or linguistic descriptions a definite structure. An expert system design of this unit is necessary in order to achieve the earlier stated goals. An expert system is a computer system employing expert knowledge to attain high levels of performance in solving the problems within a specific domain area [8].

\section{Literature Review}

For effective performance and operation of the crude oil distillation column, competent design of the CDU that improve fuel properties, increase the yields of the distillate products and meet environmental specifications is very important. Traditional systems of crude oil distilla- 
tion column design, optimization and control by previous researchers are discussed in this section. Manley [9] designed crude oil distillation column through the use of improved process thermodynamics which was also used by Santana et al. [10] to assess and improve the energy efficiency of a crude-oil tower. They were limited to the fact that the improved process designs were unproven, projected capital cost reductions were uncertain and energy savings at current prices would not justify the investment needed for process development and commercialization. The design of crude oil distillation column through the use of on-line soft-sensor for its control and optimization was discussed by Yu et al. [11] but they did not consider the design for offline application.

The use of neural network architectures to design refinery crude distillation column for the prediction of product quality was proposed by Bawazir et al. [12]. However, the design of crude oil distillation column for optimization using the neural network was left out. Liau et al. [13] picked up the challenges by using neural network for the expert system design of a crude oil distillation unit for process optimization. Torgashov [14] designed self-optimizing control of complex crude distillation column control using non-linear process model-based method. Khairiyah et al. [15] used the method proposed by Bawazir et al. [12] for design of crude oil distillation column for real time optimization. Gadalla et al. [16] used short-cut models for retrofit design of distillation columns and heat exchanger networks but were limited to the fact that short-cut models were not detailed enough and require longer time for convergence. The stage-wise modelling procedure approach was used for the design and optimization of a refinery crude distillation unit in the context of total energy requirement by Okeke et al. [17]. However, they failed to give full practical validation of the design method. Domijan et al. [18] presented a model that used the crude true boiling point curve and other routinely made laboratory measurements on crude distillation unit (CDU) for product properties prediction.

Another system of crude oil distillation column design was proposed by Macías-Hernández et al. [19] using evolving Takagi-Sugeno fuzzy models for the design of soft sensor for predicting crude oil distillation side streams. However, future research need to be directed at practical implementation of this technique in an online estimator in closed loop. Zalizawati [20] designed a continuous distillation column through development of multiple-input multiple-output (MIMO) and multiple-input single-output (MISO) neural network models. Haydary et al. [21] proposed steady-state and dynamic simulation of crude oil distillation using ASPEN PLUS and ASPEN DYNAMICS. Kanthasamy [22] used Hammerstein model and nonlinear autoregressive model with exogenous input (NARX) in designing nonlinear model predictive control (NMPC) of a distillation column. He included the activity and fugacity calculations in the model in order to account for the non-ideality of the system. Smith et al. [23] proposed design of heat integrated distillation system. However, their work was not detailed enough as the real practical analysis of the system was not stated. Tonnang et al. [3] developed a powerful controller that allows perfect control of a complex crude oil distillation column using neural network. Kansha et al. [24] investigated the crude oil distillation design with exergy analysis and the feasibility for application of the self-heat recuperation technology. The simulation was conducted by PRO/II Ver.8.1 (Invensys, Simsci) to calculate the energy required. However, this design required modification such as a self-heat exchange and an additional furnace heater for the first distillation column and no integration for hot charge method.

\section{Research Methodologies}

\subsection{Design and Control of CDU Using ANN}

Artificial neural network (ANN) models are black box models, consisting of layers of nodes with nonlinear basis functions and weighted connections that link the nodes. The inputs to the model are mapped to the outputs after being trained with a set of training or learning data to optimise the weights and biases of the nodes. Multilayer feedforward ANN (Figure 4) were mathematically proven to be a universal approximator [25]. The back propagation algorithm proposed by Rumelhart et al. [26] is the effective means for training or learning the set of data in order to optimize the weights. It is an approximate steepest descent algorithm in which performance index is mean square error [27]. Figure 5 gives the implementation procedure for the ANN. The error function of the output is given by:

$$
\text { error }=\frac{1}{2} \sum_{j=1}^{m}\left[d_{j}(n)-y_{j}(n)\right]
$$

where $d_{j}=$ Practical data of jth output neuron;

$y_{j}=$ Computed data of $j$ th ouput neuron;

$m=$ Neuron number;

$n=$ Training step.

$$
y_{k}=F_{N}\left(Z_{k}\right)=\frac{1}{1+\exp \left(-Z_{k}\right)}
$$

where $y_{k}=F_{N}\left(Z_{k}\right)=$ sigmoidal transfer function, $Z_{k}=$ sum of the jth input to the neuron multiplied by their respective weights

$$
Z_{k}=\sum_{j} w_{k j} x_{j}
$$

where $w_{k j}=$ weight of the jth input to the kth neuron of the output layer; 


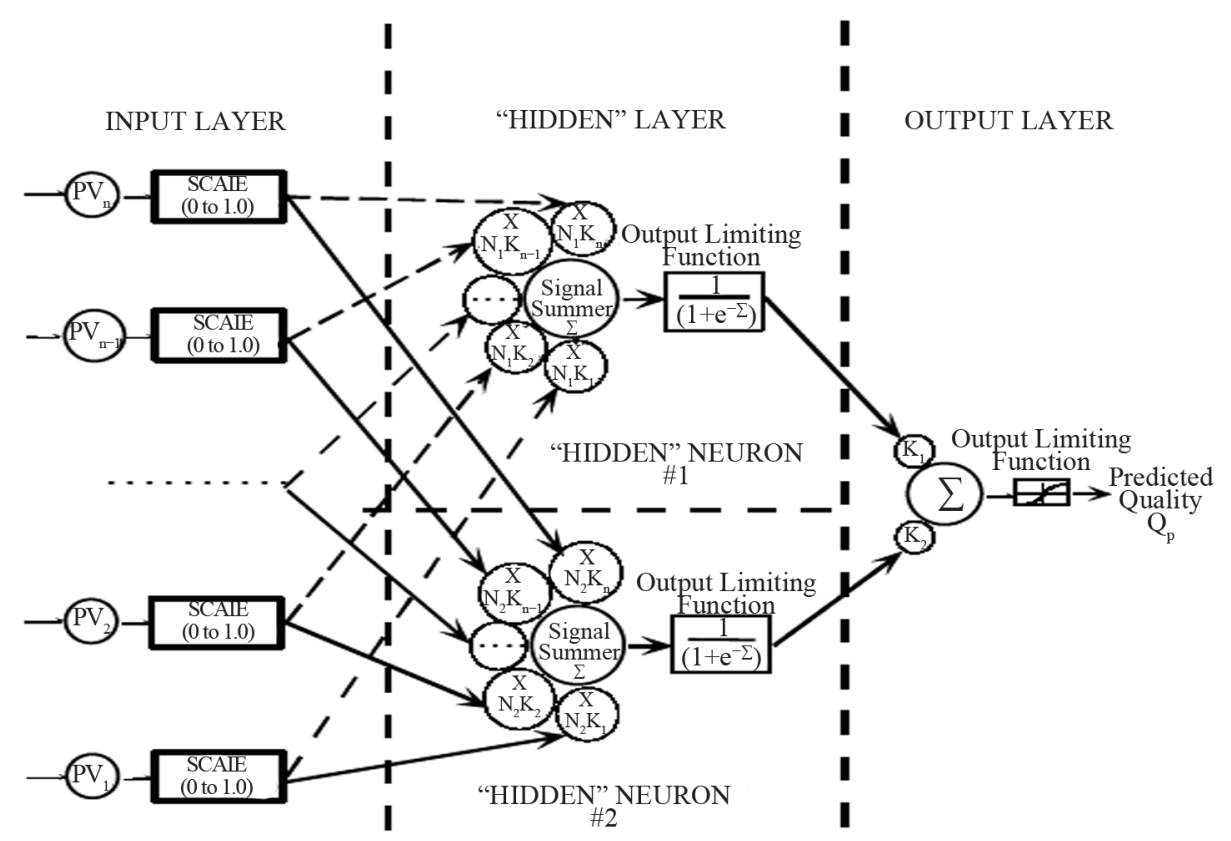

Figure 4. Multilayer feedforward ANN.

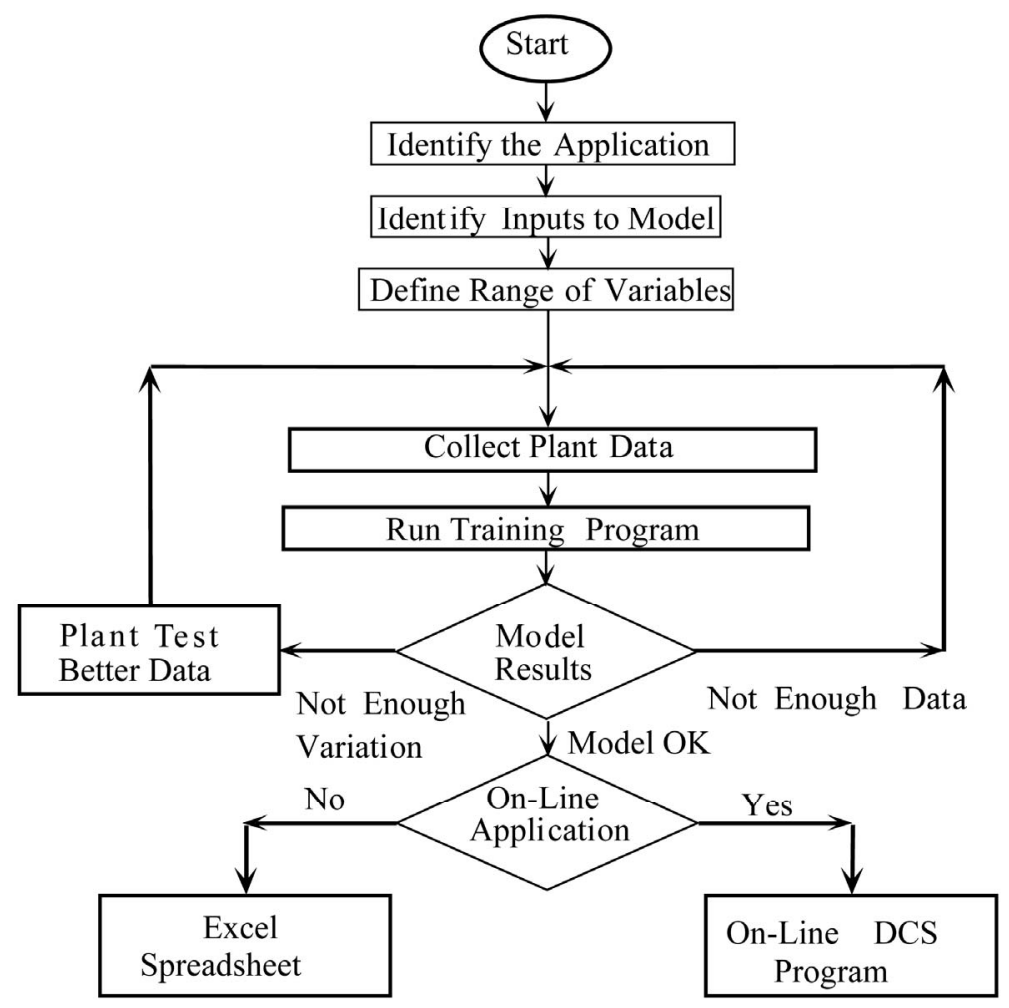

Figure 5. ANN implementation flow chart.

$x_{j}=j$ th input to the neuron.

$$
w_{i j}^{k+1}=w_{i j}^{k}+\eta \delta_{j}^{k} I_{i} f^{\prime}(s)
$$

where $w_{i j}^{k+1}=$ Weigths of the connection from unit $i$ in layer $k+1$ to unit $\mathrm{j}$ in layer $k+2$;

$w_{i j}^{k}=$ Weigths of the connection from unit $i$ in layer $k$ to unit $j$ in layer $k+1$;

$\eta=$ Learning rate constant;

$\delta_{j}^{k}=$ Signal error;

$I_{i}=$ Input vector to the networks;

$f^{\prime}(s)=$ Derivative of the networks sigmoidal transfer function; 
$s=$ Sum of all the weigths.

The development of neural network that could be used for the control of the crude oil distillation column is also discussed in this section. The tower or column receives crude oil and steam flow as inputs. Naphthalene, Kerosene, Light Diesel Oil and Heavy Diesel Oil are its outputs. Stripping (distillate flows) is sent to the storage tank, while some quantity of Naphthalene, Kerosene and Light Diesel Oil (reflux flows) are returned into the column. The input values to the neural network controller (NNC) are: distillate flows, feed flow, feed temperature, top temperature, bottom temperature, bottom composition, reflux temperature, and the tower pressure. Its output values are used to adjust the reflux flows and steam flow. The neural network controller flow chart is presented in Figure 6.

\subsection{Optimization of CDU Using Genetic Algorithm and Fuzzy Logic}

Genetic Algorithm is a powerful optimization technique based on the principles of natural evolution and selection. In the specific case of selecting the optimum set of inputs from a larger set, GA can be used to search through a large number of input combinations with interdependent variables in the artificial neural network to be designed for the crude oil distillation column. Figure 7 gives the standard structure of genetic algorithm.

Fuzzy theory is another powerful tool in the exploration of complex problems because of its ability to determine outputs for a given set of inputs without using a conventional, mathematical model. The development of fuzzy theory came from the inability to describe some physical phenomena with the exact mathematical models dictated by more conventional Boolean models. Fuzziness describes event ambiguity. It measures the degree to which an event occurs, not whether it occurs. In its simplest form a fuzzy logic is simply a set of rules describing a set of actions to be taken for a given set of inputs. It is easiest to think of these rules as if then statements of the form if $\{$ set of inputs\} then $\{$ outputs\}.

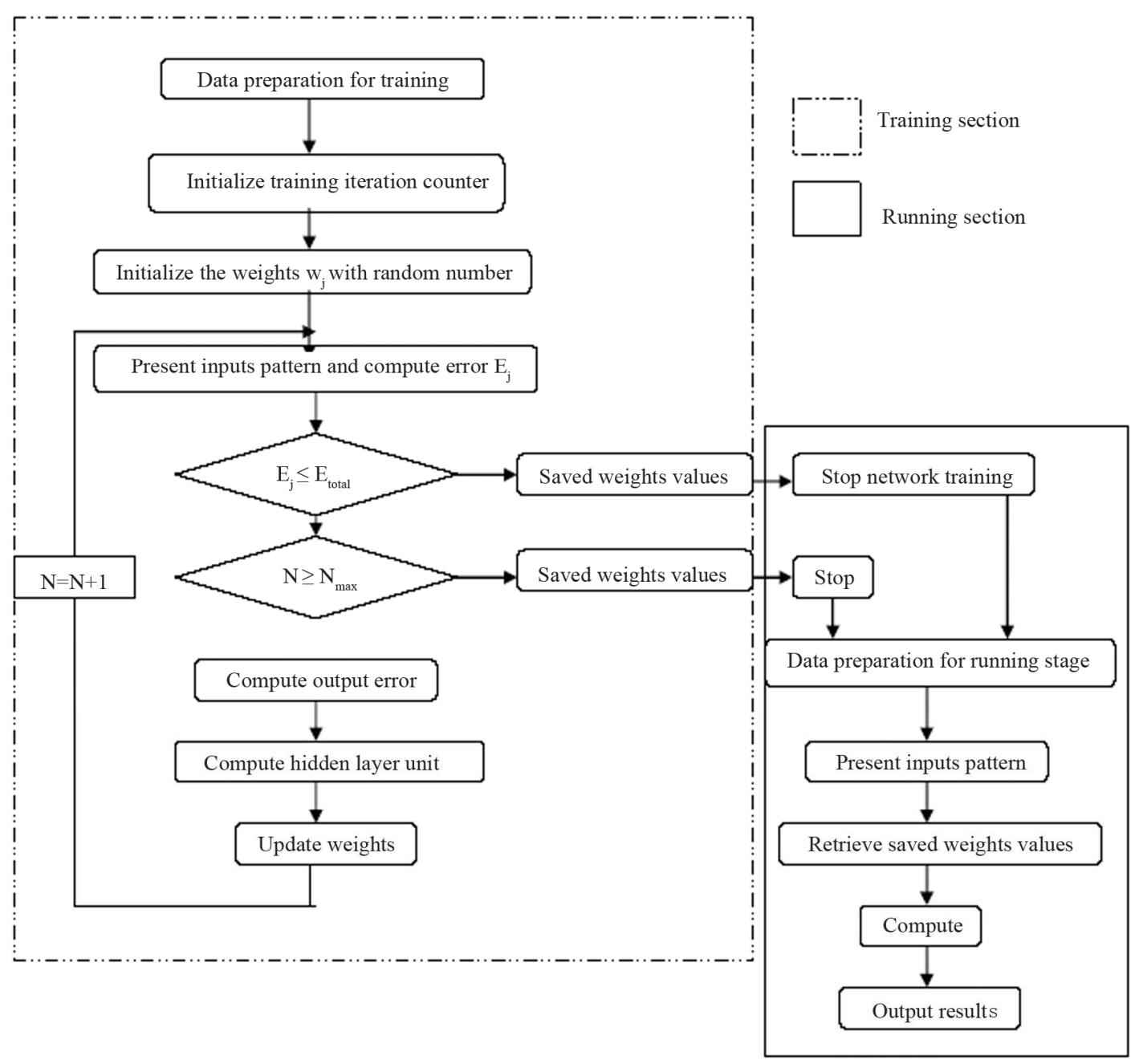

Figure 6. Neural network controller flow chart. 


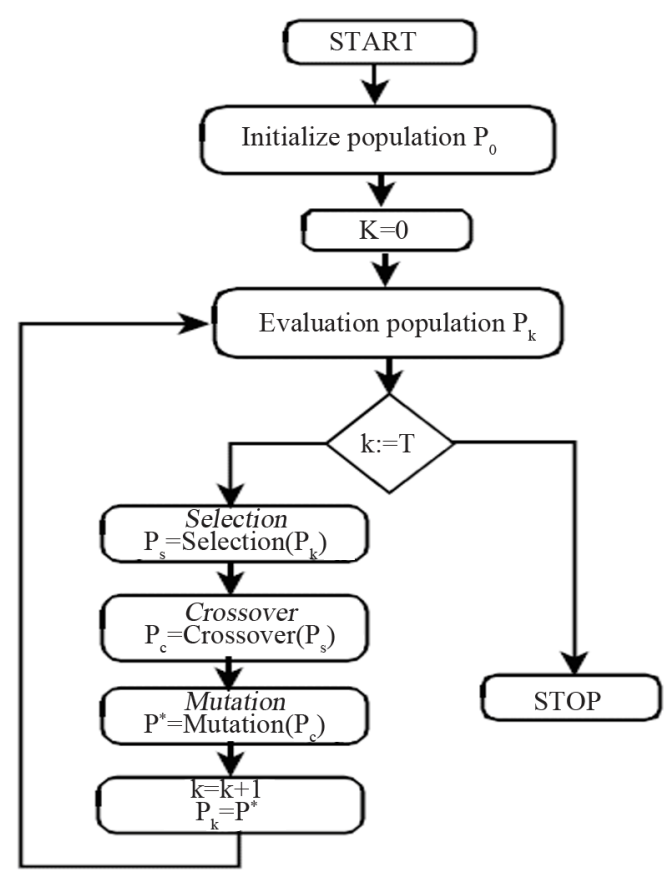

Figure 7. Standard genetic algorithm.

\section{Discussion}

After adequate scrutiny of various traditional systems of crude oil distillation column design, optimization and control, artificial neural network, fuzzy logic and genetic algorithm had been found as the best methodologies based on some established facts. They serve as substitutes for dynamic mathematical models as they are time independent. Many researchers used rigorous simulations which led to convergence problems and were also time consuming. These soft computing methodologies use error minimal method to replace the convergence problem. Also, artificial neural network models, fuzzy logic and genetic algorithm approach had been found as the effective ways to model complex processes due to their non-linear characteristic structures. Lastly, the proposed methodologies can remarkably enhance the regulatory and advanced control capabilities of various industrial processes such as crude oil distillation columns in refineries.

\section{Conclusion}

An expert system design of crude oil distillation column can be done using the artificial neural networks. The product quality specification and the optimal operation can be reached through the use of artificial neural network. Also, the crude oil distillation column optimization can be achieved using both the fuzzy logic and genetic algorithm frame work. The continuous evaluation and adjustment of process operating conditions to optimise economic productivity can be reached by these method- ologies. A neural network controller can be designed for crude oil distillation column. The developed neural network controller is capable of mapping the interactions and nonlinear dynamics of the process. Artificial neural networks, fuzzy logic and genetic algorithm framework are the best soft computing methodologies for both the expert design and optimization of crude oil distillation column. The design of neural network controller for the crude oil distillation column is also recommended in order to meet the requirements with respect to environment, health and safety of the plant personnel and the quality of the finished products.

\section{REFERENCES}

[1] K. H. Bawazeer, "Prediction of Crude Oil Product Quality Parameters Using Neural Networks," MS Thesis, Florida Atlantic University, Boca Raton, 1996.

[2] W. Heather, "Refining Crude Oil," The New Zealand Refining Company Ltd., Ruakaka, 2003.

[3] Z. E. H. Tonnang, "Distillation Column Control Using Artificial Neural Networks," M.Sc Thesis, Microprocessors and Control Engineering, Department of Electrical and Electronics Engineering, Faculty of Technology, University of Ibadan, Ibadan, 2010.

[4] S. Christos and S. Dimitrios, "Introduction to Artificial Neural Network,” Research Report, 2001.

[5] H. L. Hoffman, D. E. Lupfer, L. A. Kane and B. A. Jensen, "Distillation Column, Basic and Advance Controls Process Control, Instrument Engineer Handbook," 3rd Edition, Butherworth Heinemann, Oxford, 1995.

[6] T. M. Gowrie and V. V. C. Reddy, "Load Forecasting by a Novel Technique Using ANN," ARPN Journal of Engineering and Applied Sciences, Vol. 3, No. 1, 2008, pp. 19-25.

[7] J. W. Sea, M. Oh and T. H. Lee, "Design Optimization of Crude Oil Distillation," Chemical Engineering Technology, Vol. 23, No. 2, 2000, pp. 157-164. doi:10.1002/(SICI)1521-4125(200002)23:2<157::AID-C EAT157>3.0.CO;2-C

[8] J. McCarthy, "Some Expert System Need Common Sense," Stanford University, Stanford, 1984.

[9] D. B. Manley, "Waste Minimization through Improved Process Thermodynamics: Crude Oil Fractionation," The University of Missouri, Rolla, 1993.

[10] E. I. Santana and R. J. Zemp, "Thermodynamic Analysis of a Crude-Oil Fractionating Process," 4th Mercosur Congress on Process Systems Engineering, Vol. 21S, 2001, pp. 523-528.

[11] J. J. Yu, C. H. Zhou, S. Tan and C. C. Hang, "An On-line Soft-Sensor for Control and Optimization of Crude Distillation Column," Research Institute of Industrial Process Control, Zhejiang University, Hangzhou, 1997.

[12] K. H. Bawazir and A. Zilouchian, "Application of Neural Networks in Oil Refineries," Proceedings of 1996 IEEE International Conference on Neural Networks, New Orleans, 11-13 May 1999, pp. 126-135. 
[13] L. C.-K. Liau, T. C.-K. Yangb and M. T. Tsaib, "Expert System of a Crude Oil Distillation Unit for Process Optimization Using Neural Networks," Expert Systems with Applications, Vol. 26, No. 2, 2004, pp. 247-255. doi:10.1016/S0957-4174(03)00139-8

[14] A. Torgashov, "Nonlinear Process Model-Based Self-Optimizing Control of Complex Crude Distillation Column," European Symposium on Computer Aided Process Engineering-11, Vol. 9, 2001, pp. 793-798.

[15] M. F. Khairiyah, K. Fakhri and L. D. Peter, "Connectionist Models of a Crude Oil Distillation Column for Real Time Optimisation," Regional Symposium on Chemical Engineering 2002, Songkla, 2002.

[16] M. Gadalla, M. Jobson and M. Smith, "Optimisation of Existing Heat - Integrated Refinery Distillation Systems," Ph.D. Thesis, UMIST, Manchester, 2002.

[17] E. O. Okeke and A. A. Osakwe-Akofe, "Optimization of a Refinery Crude Distillation Unit in the Context of Total Energy Requirement," APACT03, 28-30 April 2003, NNPC R\&D Division, Port Harcourt, 2003.

[18] P. Domijan and D. Kalpić, "Off-Line Energy Optimization Model for Crude Distillation Unit," Ph.D. Thesis, Department of Applied Mathematics, Faculty of Electrical Engineering and Computing, University of Zagreb, Zagreb, 2004.

[19] J. J. Macías-Hernández, P. Angelov and X. Zhou, "Soft Sensor for Predicting Crude Oil Distillation Side Streams Using Evolving Takagi-Sugeno Fuzzy Models. Results Outlined," Proceedings of 2nd International Symposium on Evolving Fuzzy Systems, 7-9 September 2008, Lake District, IEEE Press, 2008, pp. 214-220.

[20] B. A. Zalizawati, "Development of Multiple-Input Multiple-
Output and Multiple-Input Single-Output Neural Network Models for Continuous Distillation Column," M.Sc Thesis, School of Chemical Engineering, Malaysia University, 2008.

[21] R. Kanthasamy, "Nonlinear Model Predictive Control of a Distillation Column Using Hammerstein Model and Nonlinear Autoregressive Model with Exogenous Input," Ph.D. Thesis, Universiti Sains Malaysia, 2009.

[22] J. Haydary and T. Pavlik, "Steady-State and Dynamic Simulation of Crude Oil Distillation Using ASPEN Plus and ASPEN Dynamics," Petroleum and Coal, Vol. 51, No. 2, 2009, p. 100

[23] R. Smith, M. Jobson, L. Chen and S. Farrokhpanah, "Heat Integrated Distillation System Design," Centre for Process Integration, School of Chemical Engineering and Analytical Science, The University of Manchester, 2010.

[24] Y. Kansha, A. Kishimoto and A. Tsutsumi, "Application of the Self-Heat Recuperation Technology to Crude Oil Distillation," Collaborative Research Centre for Energy Engineering, Institute of Industrial Science, The University of Tokyo, Tokyo, 2011.

[25] K. Hornik, M. Stinchcombe and H. White, "Multilayer Feedforward Neural Networks Are Universal Approximators," Neural Networks, Vol. 2, No. 5, 1989, pp. 359 366. doi:10.1016/0893-6080(89)90020-8

[26] D. Rumelhart and J. McClelland, "Parallel Distributed Processing," MIT Press, Cambridge, 1986.

[27] G. Daniel, "Principles of Artificial Neural Networks," Advanced Series in Circuits and Systems, Vol. 51, No. 2, 2007, pp. 100-109. 